\title{
Origins of Gliogenic Stem Cell Populations Within Adult Skin and Bone Marrow
}

\author{
David P. Hunt, ${ }^{1,2}$ Marija Sajic, ${ }^{2}$ Helen Phillips, ${ }^{3}$ Deborah Henderson, ${ }^{3}$ Alastair Compston, ${ }^{1}$ \\ Kenneth Smith, ${ }^{2}$ and Siddharthan Chandran ${ }^{1,4}$
}

The generation of Schwann cells from precursors within adult skin and bone marrow is of significant clinical interest because of the opportunities for disease modelling and strategies for remyelination. Recent evidence has suggested that glial cells can be generated from (i) mesenchymal stem cells (MSCs) within adult bone marrow and (ii) skin-derived precursor cells (SKPs) within adult skin. However, there is a need to clarify the developmental mechanism whereby such multipotent adult stem cell populations generate glia. We used Wnt1-Cre/ Rosa26R ${ }^{\mathrm{LacZ}}$ and Wnt1-Cre/Rosa26R ${ }^{\mathrm{YFP}}$ neural crest reporter mice to test the hypothesis that (i) MSCs and (ii) SKPs represent adult gliogenic precursor cells of neural crest origin. We demonstrate that, although labeled cells can be identified within long bone preparation, such cells are rarely found in marrow plugs. Moreover, we did not find evidence of a neural crest origin of bone marrow-derived MSCs and were not able to provide a developmental rationale for the derivation of glial cells from MSCs using this approach. In contrast, we provide robust evidence for the neural crest origin of SKPs derived from adult skin. These precursor cells reliably generate cells with a Schwann cell phenotype, expressing appropriate transcription factors and Schwann cell markers. We demonstrate multiple anatomical origins of gliogenic SKPs within adult skin. We conclude that SKPs, rather than bone marrow-derived MSCs, represent a more defined and developmentally rational source for the study and generation of Schwann cells from readily accessible adult tissues.

\section{Introduction}

$\mathrm{T}$ HE ABILITY TO GENERATE Schwann cells and their precursors from adult non-neural tissues such as bone marrow and skin is of significant clinical interest (1-3). Schwann cells can provide beneficial therapeutic effects in both the peripheral and central nervous systems by remyelination, provision of trophic support, and a role in promoting axon regeneration $(4,5)$. Furthermore, improved understanding of Schwann cell differentiation from neural crest precursor cells may provide mechanistic insight into patients with genetic disorders of neural crest and Schwann cell differentiation such as those with Shah-Waardenburg syndrome (6) and subtypes of Charcot-Marie-Tooth disease (type 1B) (7).

Schwann cells of peripheral nerves are derived from the neural crest (5). This is a transient embryonic structure, unique to vertebrates, that originates at the neural folds early in development (8). Neural crest cells migrate throughout the body where they give rise to a wide range of cells including peripheral neurons and glia, smooth muscle cells, craniofacial mesenchyme, and autonomic neurons (9).

A number of recent reports have suggested that cells with a glial phenotype can be generated from stem cells derived from both bone marrow and skin (1,10-13). However, the identity of the original cells that give rise to putative Schwann cells remains poorly defined. In both cases, it has been proposed that Schwann cells are generated from precursors within these tissues. In the case of bone marrow, Schwann cell differentiation has been reported from mesenchymal stem cells (MSCs), also termed mesenchymal stromal cells (1,11). MSCs are generated from bone marrow

\footnotetext{
${ }^{1}$ Anne Maclaren Laboratory for Regenerative Medicine, MRC Centre for Stem Cell Biology and Regenerative Medicine, Forvie Site, University of Cambridge, Cambridge, United Kingdom.

${ }^{2}$ Department of Neuroinflammation, University College London, London, United Kingdom.

${ }^{3}$ Institute of Human Genetics, Newcastle University, Newcastle upon Tyne, United Kingdom.

${ }^{4}$ Euan MacDonald Centre, Chancellors Building, University of Edinburgh, Edinburgh, United Kingdom.
} 
aspirates and are defined by their plastic adherence in serum-containing media, their cell surface marker profile, and their ability to generate mesenchymal progeny $(14,15)$. However, such reports of "transdifferentiation" have been controversial (16) and the developmental rationale for such observations lacking.

In the case of skin, Schwann cell differentiation has been reported from skin-derived precursor cells (SKPs). SKPs are defined as multipotent precursor cells that can grow as self-renewing precursors under substrate-free conditions in media specialized for neural precursor propagation (17). SKPs have also been shown to possess mesenchymal as well as neuroglial potential (18).

The developmental origins of both MSCs and SKPs have proved difficult to define, partly because they are defined by their in vitro rather than in vivo behavior. This problem is compounded by significant variation in cell culture protocols between laboratories (19). Given that Schwann cells derived from MSCs and SKPs are being considered for therapeutic purposes $(1,20)$, there is a need to provide a developmental and anatomical rationale for such claims of Schwann cell differentiation from these precursor cells. This need is highlighted by recent reports that demonstrate that minor differences in cell culture methodologies between laboratories may lead to drastically different clinical outcomes in a transplantation model of remyelination $(1,19)$.

The reported Schwann cell differentiation from MSCs and SKPs raises the hypothesis that these cells represent tissue-resident adult neural crest precursor cells. This hypothesis can be tested directly using compound transgenic mice that express reporter genes within neural crest-derived cells (21). Fate mapping experiments in Wnt1-Cre, P0-Cre, and htPA-Cre reporter mice have allowed a more detailed anatomical understanding of the mammalian neural crest contribution to various tissues including bone marrow and skin (18,21-23). In Wnt1-Cre/ Rosa26R compound transgenic animals, neural crest cells that transiently express Wnt1 during embryogenesis are permanently labeled with either a YFP (Wnt1-Cre/Rosa26R ${ }^{\text {YFP }}$ ) or $\beta$-galactosidase reporter (Wnt1-Cre/Rosa26R ${ }^{\text {LacZ }}$ ) (24).

In this article, we have examined the neural crest origins and Schwann cell potential of MSCs and SKPs using Wnt1Cre/Rosa26R ${ }^{\text {LacZ }}$ and Wnt1-Cre/Rosa26R ${ }^{\text {YFP }}$ neural crest reporter mice. Using this system we identify a possible neural crest contribution to adult long bones preparations, but demonstrate that such cells are only rarely found in bone marrow, from which MSCs are typically generated. Using this approach, we are not able to provide evidence of a neural crest origin of bone marrow-derived MSCs, nor demonstrate glial differentiation of MSCs.

In contrast, we provide robust evidence of a neural crest origin of SKPs and demonstrate multiple anatomical origins of these cells. We demonstrate reliable Schwann cell differentiation from SKPs with expression of appropriate transcription factors and Schwann cell markers.

\section{Materials and Methods}

\section{Wnt1-Cre compound transgenic mice}

Wnt1-Cre/Rosa26R ${ }^{\mathrm{Lac} Z}$ mice were generated as previously described (25). Animals were maintained according to the Animals (Scientific Procedures) Act 1986, United Kingdom, and all the experiments were approved by the Ethical Review
Committee of Newcastle University and Institute of Child Health, University College London. In brief, Wnt1-Cre and Rosa26R ${ }^{\mathrm{Lac}}$ mice were inter-crossed to produce litters in which the cells derived from the neural crest stained blue following a reaction with X-Gal (24). Pups were genotyped for the presence of the Wnt1-Cre allele. Genomic DNA was extracted from ear clips and amplified by PCR using the following primers; CGT TTT CTG AGC ATA CCT GGA and ATT CTC CCA CCG TCA GTA CG with an annealing temperature of $60^{\circ} \mathrm{C}$. The presence of a 500-bp band indicated the presence of the Wnt1-Cre allele. To generate Wnt1-Cre/Rosa26R ${ }^{\mathrm{YFP}}$ mice, Wnt1-Cre mice were crossed with Rosa26R ${ }^{\mathrm{YFP}}$ mice, which carry an EYFP expression cassette at the Rosa26 locus such that YFP expression is activated by Cre-mediated removal of a STOP cassette (26). Tissue was harvested from adult mice $>12$ weeks of age. Mice positive for both transgenes were identified by genotyping for Wnt1-Cre followed by identification of transgene-positive cells (either LacZ or YFP) in the whisker follicle as previously described (25). Littermates negative for the Wnt1-Cre allele were used as negative controls. All experiments with animal tissue were performed in accordance with UK guidelines on the care and use of experimental animals.

\section{Bone marrow cell preparation and MSC culture}

Hindlimbs of transgenic mice were isolated and the pelvis, femur, and tibia were removed. Bones were washed in Hank's buffered saline solution (HBSS) and cleaned of adherent tissue. For whole bone preparations, leg bones were cut into pieces using fine scissors and bone fragments crushed. The resulting bone suspension was incubated in $0.25 \%$ trypsin/ethylenediaminetetraacetic acid (EDTA) for $1 \mathrm{~h}$ before addition of $20 \%$ fetal calf serum (FCS). The bone preparation was triturated using a 5-mL pipette and filtered using a 40- $\mu \mathrm{m}$ cell filter (BD Biosciences, San Diego, CA). Fluorescence-activated cell sorting (FACS) analysis was performed on the resulting single cell suspension.

Bone marrow MSC cultures were derived from the bone marrow plugs of pelvis, tibia, and femurs of transgenic mice. Bones were isolated and cleaned and both ends of long bones were cut away with fine scissors. Marrow plugs were flushed from the long bones as previously described using HBSS (19). The resulting cell suspension was collected and cells counted.

MSC cultures were based on a standard and widely used protocol to isolate murine MSCs (27). Cultures were initiated by plating the cells in 6-well plates on tissue culture plastic in a plating media of Dulbecco's modified Eagle's medium (DMEM; Gibco Grand Island, NY; Invitrogen, Carlsbad, CA) $+10 \%$ FCS. The medium was changed at $24 \mathrm{~h}$ and nonadherent cells removed. To reflect variation in MSC culture methodology $(1,11)$, additional experiments using different media for MSC isolation were also performed with the following plating media: (i) DMEM+ 20\% FCS and (ii) DMEM $+10 \%$ FCS + EGF 20 ng/mL + FGF2 40 ng/mL (R\&D Systems, Minneapolis, MN). Colony-forming unit-fibroblast (CFU-F) analysis was performed as previously described (28).

Identifiable colonies were fixed at 14 days with $0.05 \%$ glutaraldehyde and incubated with X-Gal reagent for $4 \mathrm{~h}$ at $37^{\circ} \mathrm{C}$. Colonies (>50 fibroblast-like cells) were counted and identified. X-Gal reagent was removed, washed 3 times in phosphate-buffered saline (PBS), visualized with crystal violet, and photographed. 
Parallel cultures were maintained in the above media. Adipogenic and osteogenic differentiation of MSCs was confirmed using differentiation for 21 days in the following media: adipogenic differentiation media: DMEM with $10 \%$ FCS, $10 \%$ horse serum (HS), $5.0 \mu \mathrm{g} / \mathrm{mL}$ insulin, $50 \mu \mathrm{M}$ indomethacin, $1 \mu \mathrm{M}$ dexamethasone, $0.5 \mu \mathrm{M}$ IBMX. Adipogenesis was confirmed by Oil Red O staining. Osteogenic media: DMEM with 10\% FCS, 10\% HS, $1 \mathrm{nM}$ dexamethasone, $20 \mathrm{mM}$ $\beta$-glycerol phosphate, $50 \mu \mathrm{M}$ L-ascorbic acid 2-phosphate. Osteogenesis and calcium deposition were confirmed by alizarin red staining (29). In all differentiation experiments, a highly characterized mouse MSC line was used as a positive control (generously provided by D. Prockop, Tulane University under NIH grant).

\section{Skin cell preparation and skin-derived precursor cell culture}

Skin-derived precursor cells were generated from the skin of adult Wnt1-Cre/Rosa26R ${ }^{\text {Lacz }}$ and Wnt1-Cre/ Rosa26R ${ }^{\mathrm{YFP}}$ mice as previously described (25). Further cultures were also established from embryonic day 16 and adult Sprague-Dawley rats $(2,25)$. In brief, whole skin was dissected from the face or back, washed in HBSS, cleaned, and cut into $1-2-\mathrm{mm}^{2}$ pieces and incubated in $0.25 \%$ trypsin/ EDTA for 20-40 min. Following the addition of serum, the skin pieces were mechanically dissociated to release single cells and filtered through a 40- $\mu \mathrm{m}$ mesh (BD Biosciences). In FACS experiments, cells were prepared for FACS analysis at this point. The cells were collected, washed, and resuspended in DMEM (Gibco, Invitrogen) and F12 supplement (Gibco, Invitrogen) in a 3:1 ratio with 2\% B27 (Invitrogen), $1 \%$ penicillin/streptomycin/fungizone, $20 \mathrm{ng} / \mathrm{mL}$ epidermal growth factor (EGF; R\&D Systems, Minneapolis, MN), and $40 \mathrm{ng} / \mathrm{mL}$ fibroblast growth factor 2 (FGF2; R\&D Systems, Minneapolis, MN), hereafter referred to as SKP proliferation medium. Fresh proliferation medium was added to cultures every 4-5 days and spheres were passaged by mechanical dissociation with a flame-polished glass pipette.

Microdissection of the vibrissal follicle was performed according to previously described protocols (25). In brief, the whisker pad of the mouse was cut free and the whisker hair follicles were plucked from the pad. The end-bulbs of the mouse whisker follicle, which contain the dermal papilla, were isolated and washed in HBSS. The epidermis and dermal sheath of the end-bulb was inverted over the end of a pair of fine forceps, exposing the dermal papilla. The dermal papilla was then cut free. The upper hair follicle structures were isolated under a dissecting microscope and cut free (18). Single cells were generated from dermal papillae (DP) and upper hair follicles by incubation of these isolated structures in $0.25 \%$ trypsin following incision with tungsten needles. Single cells were either cultured in SKP proliferation medium or plated directly onto coverslips coated in polyD-lysine/laminin and differentiated as described below in Schwann cell differentiation medium.

For Schwann cell differentiation, papilla spheres were plated on glass coverslips coated with poly-D-lysine and laminin. Spheres were adhered overnight in SKP proliferation medium with the addition of $10 \%$ FCS. Following adherence and outgrowth of cells, the medium was replaced with Schwann cell differentiation medium that consisted of a basal medium of DMEM/F12 (3:1 ratio), 2\% B27, 1\% FCS.
To this basal medium, $4 \mu \mathrm{M}$ forskolin (Sigma, St. Louis, MO) and $50 \mathrm{ng} / \mathrm{mL}$ Neuregulin-1 (R\&D Systems, Minneapolis, $\mathrm{MN}$ ) were added. Cells were differentiated for 10-21 days with $50 \%$ replacement of differentiation medium every 4 days.

\section{Immunohisto- and immunocytochemical analysis}

Facial skin and whisker pad for immunohistochemical analysis were fixed in $4 \%$ paraformaldehyde, immersed in $30 \%$ sucrose, mounted in OCT compound (BDH; Dorset, $\mathrm{UK})$ and frozen at $-20^{\circ} \mathrm{C}$. Blocks were sectioned at $14-\mu \mathrm{m}$ intervals using a Leica cryostat (Heerbrugg, Switzerland).

Long bones were decalcified in $0.5 \mathrm{M}$ EDTA for $48 \mathrm{~h}$ before further processing. Sections for X-Gal analysis were similarly processed but were fixed in $0.05 \%$ glutaraldehyde. For X-Gal staining, cells, vibrissal follicles, and small sections of skin were fixed as above and incubated with X-Gal (Imgenex) $40 \mathrm{mg} / \mathrm{mL}, 0.1 \mathrm{M}$ potassium ferricyanide, and 0.1 $\mathrm{M}$ potassium ferrocyanide with $1 \mathrm{M}$ magnesium chloride for $4 \mathrm{~h}$.

For immunocytochemistry experiments, cells were fixed in fresh $4 \%$ paraformaldehyde for $15 \mathrm{~min}$ and washed in PBS. Cells were permeabilized and blocked for $1 \mathrm{~h}$ with PBS, $0.1 \%-0.3 \%$ Triton-X, and 5\% normal goat serum. Primary antibodies were diluted in PBS and incubated overnight at $4^{\circ} \mathrm{C}$. Primary antibodies used were: fibronectin (rabbit polyclonal, 1:800; Sigma), nestin (mouse IgG1, 1:200; Chemicon MAB353), ßIII-tubulin (mouse IgG2b, 1:500; Sigma), glial fibrillary acidic protein (GFAP, 1:250; DAKO), S100 (rabbit polyclonal, 1:200; DAKO), p75 (mouse IgG1 monoclonal, 1:50; Chemicon MAB365R), CNPase (mouse IgG1 monoclonal, 1:100; Sigma C5922), Sox10 (rat:monoclonal mouse IgG1, 1:50; R\&D Systems MAB2864. mouse:rabbit polyclonal, 1:500; Abcam AB27655). Secondary antibody (Alexa Fluor, 1:1,000; Invitrogen, Carlsbad, CA) was applied for $1 \mathrm{~h}$ at $37^{\circ} \mathrm{C}$ in PBS/Hoechst $(1: 5,000)$. Cells were viewed under a Leitz microscope with appropriate filters for cell identification. Schwann cell cultures derived from juvenile sciatic nerve were established from rat according to standard culture protocols as immunocytochemical and morphological positive controls.

\section{FACS analysis and sorting}

FACS analysis and sorting were performed on a 3 laser CyAn (Beckman Coulter). For all FACS analysis of tissue from Wnt1-Cre/Rosa26R ${ }^{\mathrm{YFP}}$ mice, negative controls using nontransgenic littermates were used. Cell sorting was performed by initial gating on FSC/SSC and then on FSC/pulse width to gate out doublets and multiples.

Bone marrow sorting. FACS sorting was performed on bone marrow aspirates from long bones and YFP+ fractions sorted into 24-well tissue culture plates at a density of 1,000 cells/ $\mathrm{mL}$. YFP+ cells were collected and plated in MSC medium containing DMEM and 10\% FCS. Nonadherent cells were removed at $24 \mathrm{~h}$. Plates were examined for adherent colonies at day 14 as described earlier.

Skin sorting. Final gating was performed on YFP+ and YFP- cells and cells sorted into SKP proliferation media. Sorted YFP+ and YFP- cells were plated into 96-well plates at a density of 1,000 cells/well. Sphere formation was assessed by counting spheres at day 14 . 
Primary spheres were passaged as described earlier. Analysis of FACS data was performed using WinMDI software.

\section{Results}

\section{Identification of YFP+ and LacZ+ cells within long bones of adult neural crest reporter mice}

Expression of $\beta$-galactosidase (LacZ) and YFP reporters was confirmed within neural crest-derived structures of embryonic and adult Wnt1-Cre/Rosa26R ${ }^{\mathrm{YFP}}$ and Wnt1-Cre/Rosa26R ${ }^{\text {LacZ }}$ mice (Supplementary Fig. 1A and 1B; Supplementary materials are available online at www .liebertonline.com/scd). In adult mice, reporter transgene expression was observed in multiple neural crest-derived structures including Schwann cells of spinal roots and sciatic nerve (Supplementary Fig. 1C-1E) (21).

Reports have suggested that Schwann cells can be generated from bone marrow-derived MSCs and recent reports have postulated a potential neural crest origin of certain MSC populations within bone $(28,30)$. We therefore examined the developmental origin of MSCs derived from bone marrow of pelvis and long leg bones of Wnt1-Cre/Rosa26R ${ }^{\text {YFP }}$ and Wnt1-Cre/Rosa26R ${ }^{\mathrm{LacZ}}$ mice.

First we identified a population of cells within adult long bones, which express reporter transgenes. Single cell suspensions were obtained from pelvis, femur, and tibia of adult mice using protocols which involved crushing of the whole long bone followed by enzymatic and mechanical dissociation to a single cell suspension (28). FACS analysis of the resulting cell suspension from whole bone preparations of Wnt1-Cre/Rosa26R ${ }^{\mathrm{YFP}}$ mice identified a rare YFP+ population of cells within adult bone (Fig. 1A and 1B). However, although YFP+ cells could be readily identified within whole bone preparations, which had undergone enzymatic dissociation $(0.022 \% \pm 0.005 \%, n=6$, approximate frequency $1: 5,000$, Figure 1B), these cells were of lower frequency in single cell preparations derived from marrow plug aspirates of long bones $(0.0008 \% \pm 0.0005 \%, n=6$, approximate frequency 1:125,000, Figure 1D).

We identified the anatomical location of transgene-positive cells within long bones using the Wnt1-Cre/Rosa26R ${ }^{\mathrm{LacZ}}$ mouse. X-Gal staining of whole long bone preparations demonstrated LacZ+ cells within a number of structures, including bone cortex, articular cartilage, and vascular endothelium
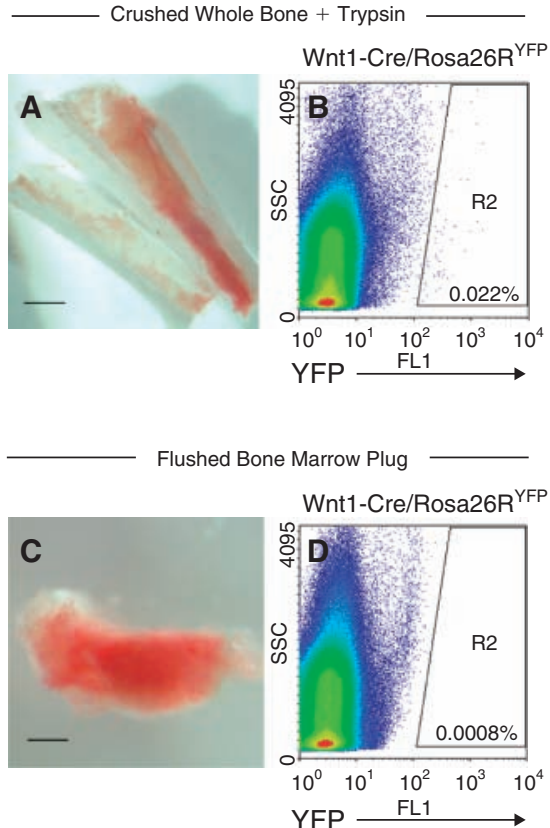
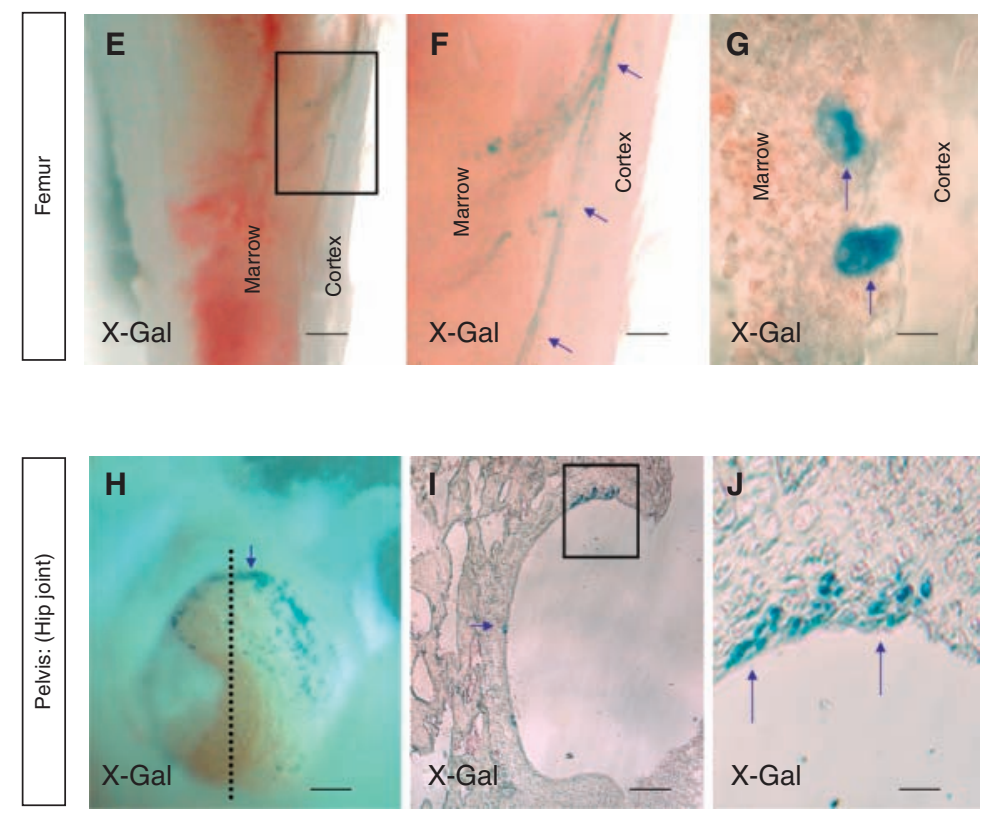

FIG. 1. Cells expressing neural crest reporter transgenes can be identified within long bone preparations of Wnt1-Cre/ Rosa26R ${ }^{\text {YFP }}$ and Wnt1-Cre/Rosa26R ${ }^{\text {LacZ }}$ mice but rarely within bone marrow plug aspirates. (A) Whole long leg bones cut open with sharp scissors, as seen under dissecting microscope. Single cells suspensions were obtained by crushing whole long bones and subsequent mechanical and enzymatic dissociation of cells. (B) Fluorescence-activated cell sorting (FACS) plot of single cell suspension obtained from crushed whole bone marrow incubated with trypsin. The $0.022 \% \pm 0.005 \%(n$ $=6$ ) of freshly isolated single cells express the YFP reporter. (C) Bone marrow obtained by aspiration of femur, seen under dissecting microscope. Single cell suspensions obtained from such bone marrow aspirates contain only rare YFP+ cells $0.0008 \% \pm 0.0005 \%(n=6)$ as determined by FACS analysis $(\mathbf{D})$, suggesting that such cells are rarely found in the bone marrow. (E) Longitudinal cross section of femur derived from Wnt1-Cre/Rosa26R ${ }^{\text {LacZ }}$ mice viewed under dissecting microscope following X-Gal staining. (F: higher magnification of inset E) X-Gal-positive cells are identified at the boundary between cortex and marrow and along blood vessels within the marrow (blue arrows). (G) Light microscope examination of decalcified long bone sections demonstrates LacZ+ cells between cortex and marrow. Such cells remain adherent to the cortex following bone marrow aspiration. $(\mathbf{H})$ LacZ+ cells are also identified within articular surface of the hip joint of the pelvis of Wnt1-Cre/Rosa26R ${ }^{\mathrm{Lac}}$ adult mice (dissection microscope: positive cells, blue arrow). (I and J) Cross section of the hip joint (dotted line in $\mathbf{H}$ ) demonstrates $\mathrm{X}$-Gal-positive cells within articular cartilage (blue arrows). 
(Fig. 1E-1G and 1H-1J). LacZ+ cells were often identified at the boundary of the marrow and bone cortex but were only rarely identified within marrow plug (Fig. 1F and 1G).

\section{No evidence of a neural crest origin of MSCs derived from marrow plug aspirates of long bones}

Although MSCs can be generated from a variety of a tissues, MSCs used for clinical purposes are typically generated from aspiration of bone marrow rather than whole bone preparations $(14,30)$. We therefore used adult Wnt1-Cre/ Rosa26R ${ }^{\mathrm{LacZ}}$ mice to examine the neural crest origins of MSCs derived from bone marrow plugs of long bones. The first step in MSC culture is the isolation of colony-forming cells that adhere to tissue culture plastic (Fig. 2A). Using a standard colony-forming assay derived from marrow aspirates of long bones (tibia, femur, pelvis) of Wnt1-Cre/Rosa26R ${ }^{\text {LacZ }}$ mice, no colonies were identified which expressed LacZ ( $n$ $=5$ mice, 3 bones per animal). Typical sequential $X-G a l$ and crystal violet staining of colonies is shown in Figure 2B, 2C, 2D, and 2E.

Parallel cultures, expanded in MSC culture conditions, underwent adipogenic and osteogenic differentiation, confirming the mesenchymal potential of these cells (Fig. 2G and 2H).

Different cell culture methodologies have been used to isolate MSCs. To reflect the methodologies used in works that report Schwann cell differentiation from MSCs, we similarly analyzed colony-forming cells in the presence of

A
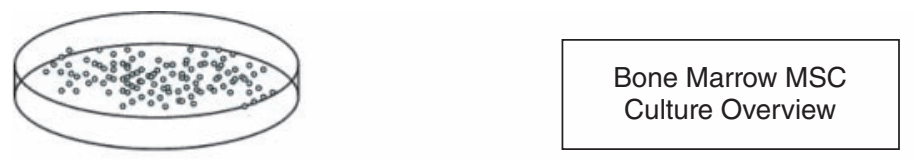

Starting Population: Bone Marrow Plug
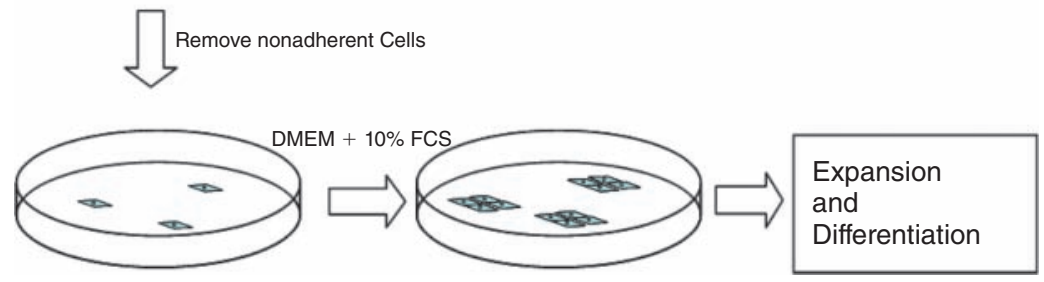

Colony-initiating Cells
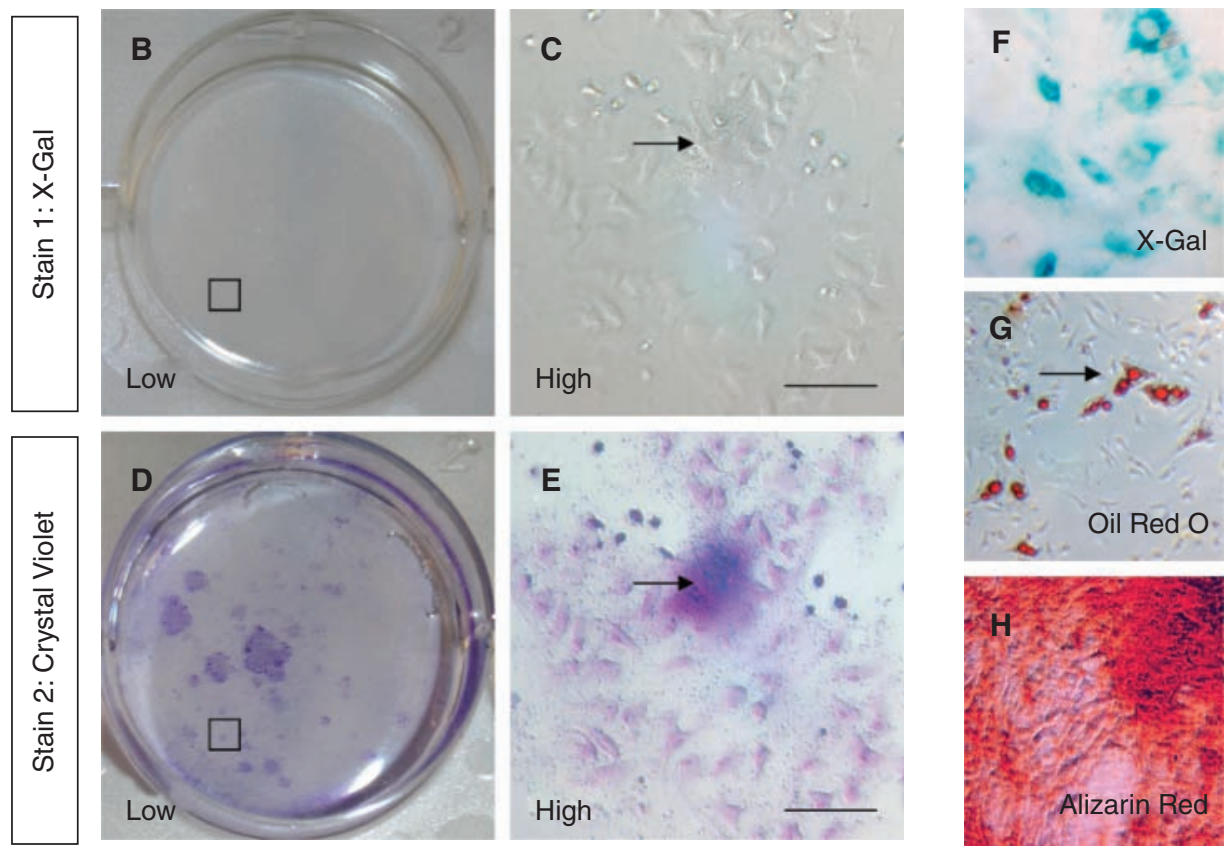

FIG. 2. No evidence of a neural crest origin of bone marrow-derived mesenchymal stem cells (MSCs). (A) Overview of MSC culture and isolation of colony-initiating cells derived from a starting population of aspirates of bone marrow. (B-E) Colony-forming units-fibroblasts (CFU-F), which subsequently form MSC cultures isolated from long bone marrow aspirates of Wnt1-Cre/Rosa26R ${ }^{\text {LacZ }}$ mice, do not express the X-Gal reporter (B and C, X-Gal staining, D and E, crystal violet staining of same well to demonstrate location of cell colonies). Encircled area $\mathbf{B}$ and $\mathbf{D}$ seen under higher power (C and $\mathbf{E})$. (F) X-Gal-positive control (whisker dermal papilla-derived fibroblasts). (G and $\mathbf{H}$ ) The adipogenic and osteogenic potential of parallel MSC cultures is confirmed by Oil Red O $(\mathbf{G})$ and alizarin red $(\mathbf{H})$ staining. 
different plating media, in particular (i) DMEM $+20 \%$ FCS (11) and (ii) DMEM + 10\% FCS + EGF $20 \mathrm{ng} / \mathrm{mL}+$ FGF 40 $\mathrm{ng} / \mathrm{mL}$ (1). All plastic adherent colonies in these conditions were negative for $\mathrm{X}-\mathrm{Gal}$ expression.

These results do not exclude the possibility that rare adult MSCs derived from bone marrow aspirates express neural crest reporter transgenes. We therefore asked whether MSCs could be cultured directly from YFP+ fraction of bone marrow. Freshly isolated FACS sorting of bone marrow allowed the prospective isolation of $\mathrm{YFP}+$ cells from whole bone marrow aspirates. These YFP+ cells were plated on tissue culture plastic in the presence of $10 \%$ FCS. No adherent colonies with MSC features could be identified (3 independent FACS sorting experiments).

Therefore, MSCs derived from bone marrow aspirates from adult long bones do not express neural crest transgenes. In addition, MSCs could not be cultured from YFP+ cells isolated from adult Wnt1-Cre/R26R ${ }^{\mathrm{YFP}}$ bone marrow. Taken together, these results argue against a neural crest origin for MSCs derived from adult long bone aspirates.

We used a variety of published protocols used to direct differentiation of MSCs into cells of a Schwann cell lineage $(11,12)$. However, we were unable to generate Sox10+/GFAP+ cells with a Schwann cell phenotype from MSCs derived from plug aspirates.

Taken together, we are unable to provide evidence for a neural crest lineage of bone marrow plug-derived MSCs or evidence that these cells behave as neural crest precursor cells with gliogenic potential.

\section{Multiple lines of evidence for a neural crest origin of skin-derived precursor cells and their Schwann cell progeny}

Skin-derived precursor cells grow as self-renewing spheres in media containing EGF and FGF2 (2). An overview

FIG. 3. Evidence of a neural crest origin of skin-derived precursor cells (SKPs) derived from adult skin. (A) Overview of SKP culture from primary tissue. Whole skin is mechanically and enzymatically dissociated to single cells and subsequently cultured in media containing EGF and FGF2 to form nestin + spheres. These spheres are subsequently plated on PDL/laminin-containing coverslips and differentiated into cells with a glial phenotype. (B) Primary spheres form in culture (C, D) SKPs differentiate in culture over a 3-4 week period to generate a subpopulation of cells with a Schwann cell morphology. (E-J) Wnt1-Cre/Rosa26R reporter expression is seen in both primary spheres and differentiated SKPs derived from back skin and facial skin of both Wnt1Cre/Rosa26R ${ }^{\text {LacZ }}$ mice $(\mathbf{E}$ and $\mathbf{H}, \mathbf{X}-$ Gal staining $=$ blue) and Wnt1-Cre/Rosa26R ${ }^{\text {YFP }}$ mice (inset $\mathbf{E}$, inset $\mathbf{H}$ : phase and YFP = green). (F and I) Following differentiation of spheres in media containing Neuregulin and forskolin, cells derived from facial and back skin-derived SKPs co-express YFP and S100. (G and J: higher magnification). Position of initial sphere plating marked (S). ( $\mathbf{K}$ and $\mathbf{L}$ ) Immunophenotype of SKP-derived Schwann cells. SKP-derived Schwann cells express S100 (red) and co-express Sox10 (green). (M and N) SKP-derived Schwann cells also express MBP (green) and S100 (red) with co-expression of Sox10 (green). of the culture methodology used to generate SKPs and their Schwann cell progeny is outlined in Figure 3A and 3B-3D. In contrast to MSCs, culture-initiating cells grow as primary free-floating spheres. Transgene expression was examined in SKPs derived from adult Wnt1-Cre/Rosa26R ${ }^{\text {YFP }}$ and Wnt1Cre/Rosa26R ${ }^{\text {Lac }}$ mice. Consistent with previous results (18), all SKPs generated from whole facial and back skin of adult

A
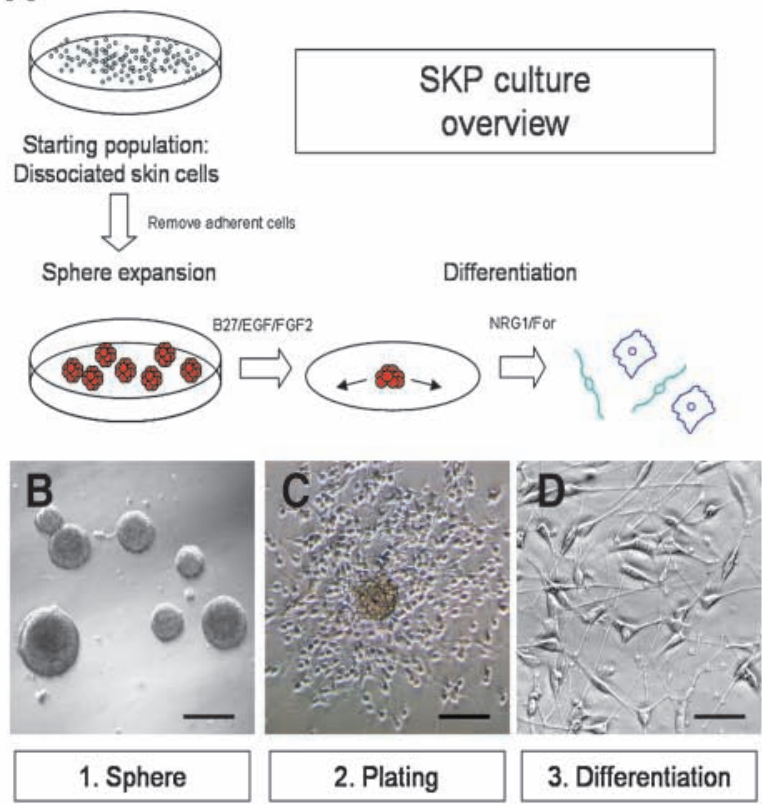

1. Sphere

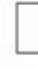

2. Plating

3. Differentiation
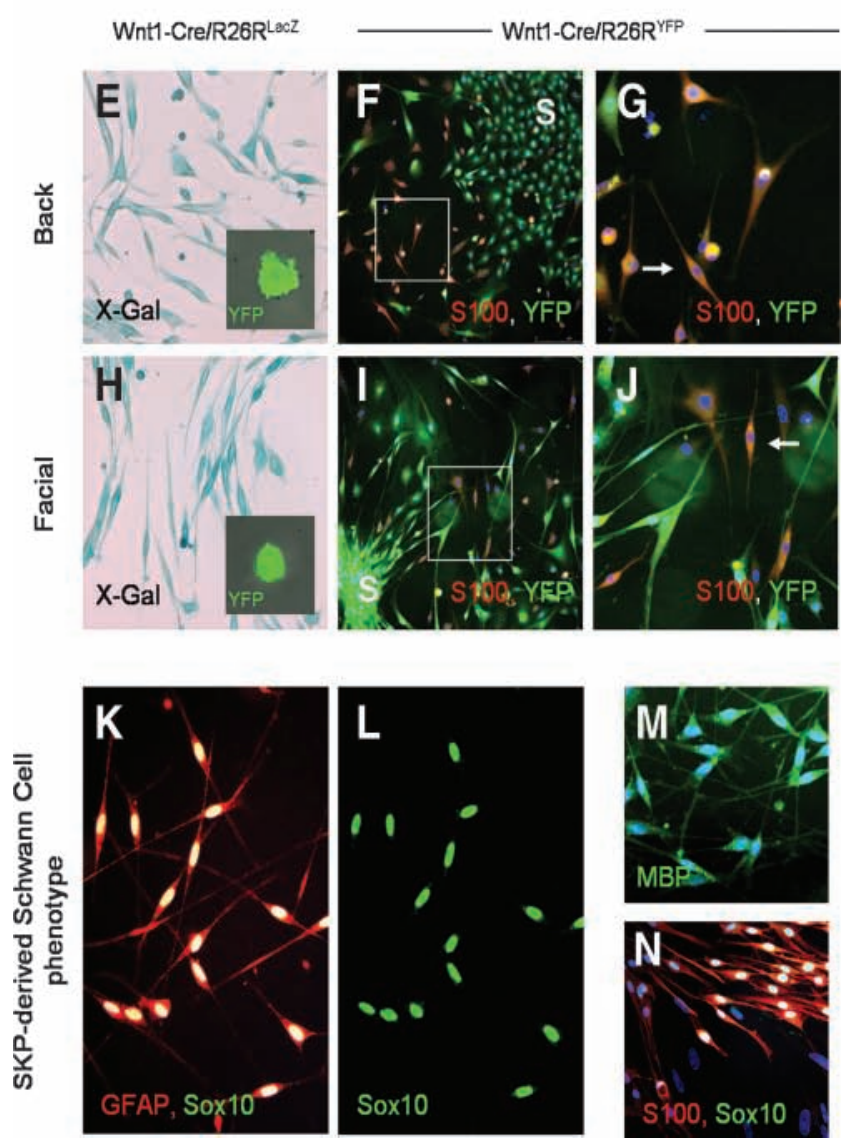
A

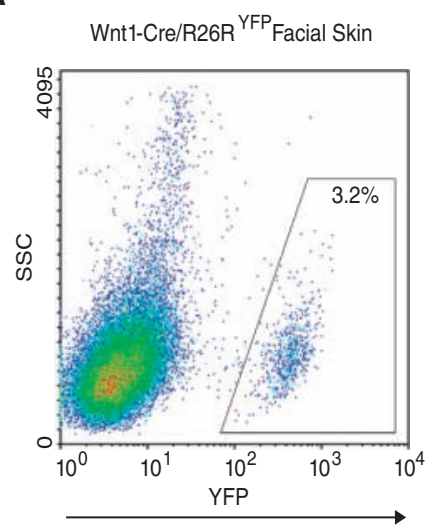

B

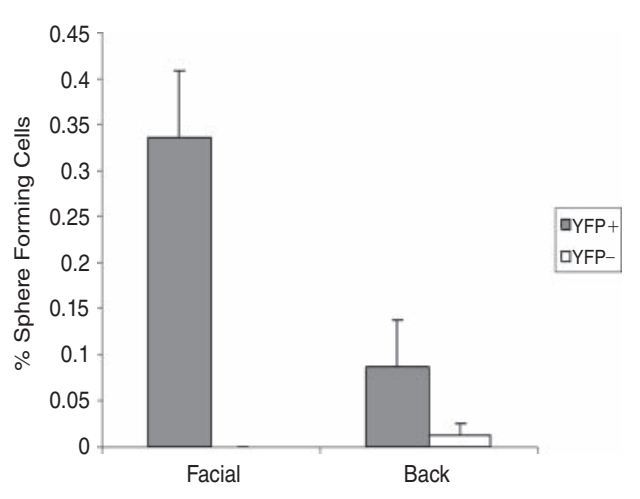

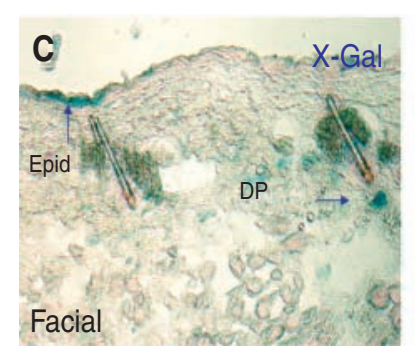
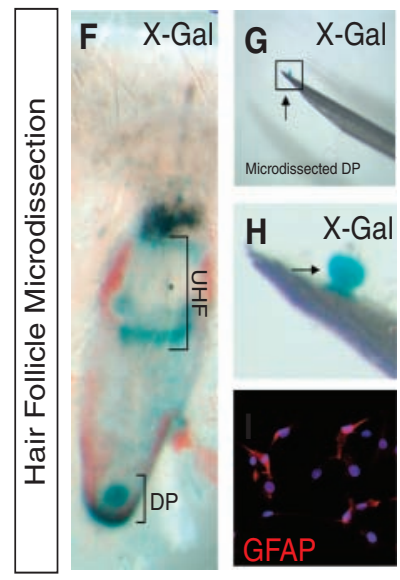
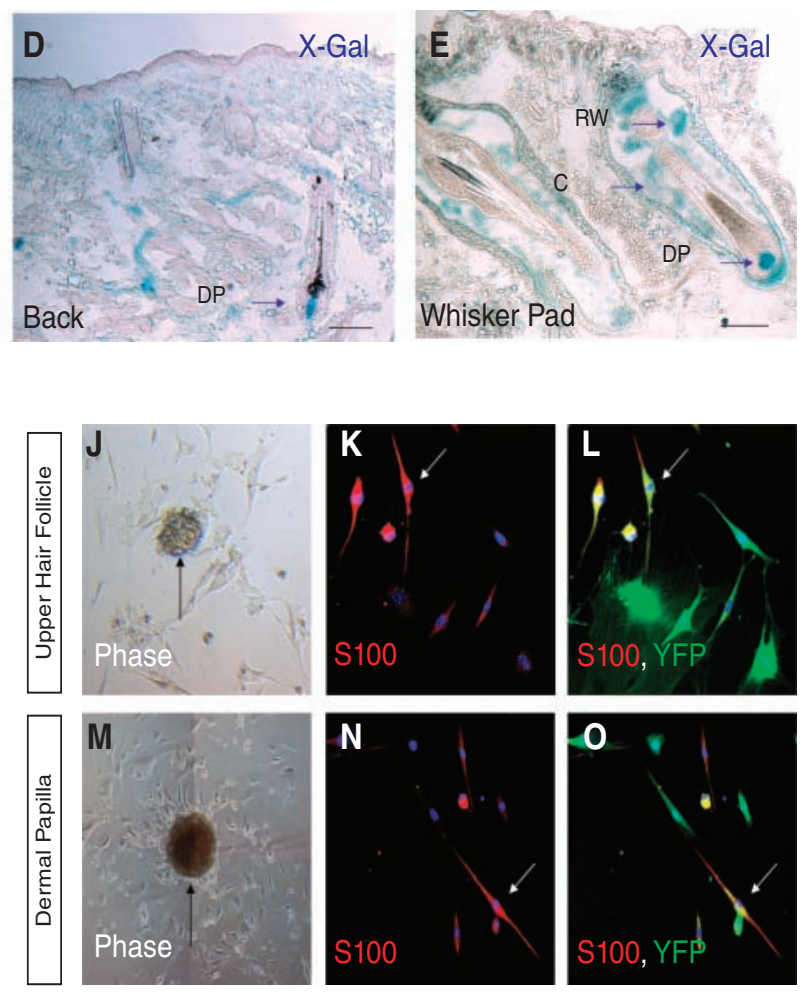

FIG. 4. Diverse anatomical origins of neural crest precursor cells derived from adult skin. (A) Fluorescence-activated cell sorting (FACS) analysis of freshly dissociated facial skin cells derived from Wnt1-Cre/Rosa26R ${ }^{\text {YFP }}$ adult facial mouse skin, demonstrating a discrete population of transgene-expressing cells that can be isolated by FACS sorting. (B) Sphere-forming cells are found predominantly in the YFP+ fraction of freshly dissociated adult skin cells derived from Wnt1-Cre/Rosa26R ${ }^{\mathrm{YFP}}$ mice (see text). (C-E) X-Gal staining of whole skin from facial, back, and whisker pad regions demonstrates widespread distribution of LacZ+ cells. (C) LacZ+ cells are identified within the epidermis (Epid), dermis, nonvibrissal hair follicle, and dermal papillae (DP) of facial skin. (D) LacZ+ cells are found only within the dermis of back skin and within hair follicles (dermal papilla shown). (E) LacZ+ cells are identified within multiple structures within vibrissal follicles, including the DP and upper hair follicle structures such as the ringwulst (RW) and other epidermal components and capsula (C). Microdissection of vibrissal hair follicles demonstrates multiple anatomical origins of gliogenic skin-derived precursor cells (SKPs). (F) X-Gal staining of a single vibrissal hair follicle from an adult Wnt1-Cre/Rosa26R ${ }^{\mathrm{LacZ}}$ mouse. The upper hair follicle (UHF) and DP are discrete hair follicle regions that contain cells of neural crest origin and are amenable to microdissection (X-Gal $=$ blue). $(\mathbf{G}$ and $\mathbf{H})$ Microdissection of X-Gal-stained hair follicle demonstrates clean isolation of dermal papilla (resting on the point of a 32G needle, blue = X-Gal staining). (I) Single cells liberated from the dermal papilla and differentiated directly in Schwann cell differentiation medium generate cells with a Schwann cell phenotype, which express GFAP (red). (J-L) Single cells generated from the microdissected upper hair follicle of adult Wnt1-Cre/Rosa26R ${ }^{\mathrm{YFP}}$ mice form YFP+ spheres (J), which are able to generate S100+/YFP+ cells with a Schwann cell phenotype (K and L, S100 red, YFP green). (M-O) Similarly single cells generated from the vibrissal dermal papilla of adult Wnt1-Cre/Rosa26R ${ }^{\mathrm{YFP}}$ mice form YFP+ spheres (M), which are able to generate S100+/YFP+ cells with a Schwann cell phenotype (N and O S100 red, YFP green). 
Wnt1-Cre/Rosa26R ${ }^{\text {YFP }}$ mice expressed YFP when cultured in standard conditions and observed under fluorescence microscopy for YFP expression (Fig. 3E and 3H inset). LacZ expression was confirmed in SKPs and their differentiated progeny derived from back and facial skin from Wnt1-Cre/ Rosa26R ${ }^{\text {LacZ }}$ mice (Fig. 3E and 3H).

SKPs derived from back and facial skin of Wnt1-Cre/ Rosa26R ${ }^{\text {YFP }}$ mice were differentiated in the presence of Neuregulin-1 and forskolin (2). In contrast to the results observed for MSCs, SKPs derived from Wnt1-Cre/Rosa26R ${ }^{\text {YFP }}$ mice reliably generated S100+/YFP+ cells with a Schwann cell morphology (Fig. 3F and 3I, and 3G and 3J). Therefore, Wnt1-Cre reporter expression was observed in both undifferentiated SKPs and their progeny from both facial and back skin. SKP-derived Schwann cells consistently expressed Schwann cell transcription factors and markers including Sox10/Krox20/p75/S100/GFAP/MBP/P0 (see Fig. 3K-3N and Supplementary Fig. 2).

We examined SKPs for expression of neural crest stem cell transcription factors. Sox10 is an important transcription factor expressed in neural crest stem cells, involved in maintenance of multipotency and differentiation to a glial phenotype (31). We observed Sox10 expression in SKPs derived from embryonic day 16 and adult rats and their Schwann cell progeny (Supplementary Fig. 2A, 2C-2E, and 2O-2T). Immediately upon plating, SKPs generated from both embryonic and adult rat and mouse skin expressed Sox10 (Supplementary Fig. 2O-2Q). In differentiated SKP progeny, maintenance of Sox10 expression was associated with differentiation to a Schwann cell phenotype $(87.16 \% \pm 3.8 \%$ Sox $10+$ cells display a S100+ Schwann cell phenotype compared with $2.7 \% \pm 2.01 \%$ Sox $10-$ cells, $n$ $=5$; Supplementary Figure $2 \mathrm{~W}$ ). The dermal papilla of the vibrissal hair follicle has previously been identified as a highly enriched niche of SKPs in adult skin $(25,32)$ and Sox10 expression was observed within the DP of adult vibrissal follicles (Supplementary Fig. 2I-2N). In addition to Sox10, S100, and GFAP expression, other neural crest transcription factors such as Krox20 and myelin proteins such as P0 and MBP were detected in the Schwann cell progeny of SKPs (Fig. 3K-3N, Supplementary Fig. 2F-2H).

\section{Heterogeneous anatomical origins of skin-derived precursor cells and their Schwann cell progeny}

We next asked whether endogenous SKPs are contained within the YFP+ fraction of freshly dissociated skin cells derived from Wnt1-Cre/Rosa26R ${ }^{\mathrm{YFP}}$ mice. FACS analysis of freshly dissociated facial skin cells identified a distinct population of transgene-expressing cells (Fig. 4A). FACS sorting of freshly dissociated facial and back skin from Wnt1-Cre/ Rosa26R ${ }^{\mathrm{YFP}}$ mice was performed and YFP+ and YFP- cell fractions were cultured in standard SKP conditions (Fig. 4A and $4 \mathrm{~B})$. In facial skin, sphere-forming cells were contained entirely within the YFP+ fraction (Fig. 4B: 0.33\% $\pm 0.073 \%$ YFP+ cells formed spheres from facial skin $n=17$ sorted cultures from 3 mice. No YFP- cells formed spheres from facial skin, $n=17$ ). Spheres could not be generated from the YFP- fraction of facial skin, although rare spheres could be generated from YFP- fraction of dissociated back skin (Fig. 4B: $0.088 \% \pm 0.05 \% \mathrm{YFP}+$ cells formed spheres from sorted back skin $n=7$ sorted cultures from 3 mice; $0.0125 \%$ $\pm 0.01 \% \mathrm{YFP}-$ cells formed spheres from sorted back skin, $n=7)$.

Given that SKPs are found within the YFP+ fraction of cells in adult skin, the anatomical distribution of YFP+ and LacZ+ cells within adult skin of transgenic mice was examined. The frequency of YFP+ cells in scalp and facial skin was determined by FACS analysis of freshly dissociated cells and was 3.06\% $\pm 1.11 \%(n=3)$ and $3.25 \% \pm 0.31 \%$ $(n=3)$, respectively. The frequency of YFP+ cells in back skin was $0.87 \% \pm 0.21 \%(n=3)$. LacZ+ cells were identified in adult skin of Wnt1-Cre/Rosa26R ${ }^{\mathrm{LacZ}}$ mice in multiple anatomical regions (Fig. 4C-4E). In facial skin, LacZ+ cells were identified in the interfollicular epidermis, dermis, and hair follicles (Fig. 4C). In back skin, LacZ+ cells were identified predominantly within the dermis and occasionally within the dermal papilla of hair follicles, although at a lower frequency to those observed in facial skin (Fig. 4D). Consistent with previous results, a high frequency of LacZ+ cells were observed in multiple structures within epidermal and dermal contributions to vibrissal follicles (Fig. 4E). LacZ expression was also seen in smaller pelage facial hair follicles, in particular, within the dermal papilla (Fig. 4C and 4E).

To examine further the anatomical origins of SKPs that could give rise to Schwann cells, we microdissected the vibrissal follicle (Fig. 4F-4H). The vibrissal follicle represents an attractive structure to microdissect given its size, heterogeneous developmental origins, and variety of cell types (18). X-Gal staining of the vibrissal follicles of adult Wnt1-Cre/ Rosa26R ${ }^{\mathrm{LacZ}}$ mice demonstrates a significant neural crest contribution to multiple regions of the vibrissal follicle, in particular, the dermal papilla and upper hair follicle (Fig. 4F). These structures could be cleanly isolated by microdissection (Fig. 4G and 4H). Microdissection of the dermal papilla and upper hair follicle of Wnt1-Cre/Rosa26R ${ }^{\mathrm{YFP}}$ mice demonstrated that YFP+ spheres could be generated from these discrete anatomical regions (Fig. 4J and 4M). Furthermore, these spheres generated S100+/YFP+ Schwann cell progeny on differentiation (Fig. $4 \mathrm{~K}$ and $4 \mathrm{~L}$, and $4 \mathrm{~N}$ and $4 \mathrm{O}$ ). GFAP+ cells with a Schwann cell phenotype could be generated from both immediately plated dermal papilla cells in addition to culture-expanded dermal papilla-derived SKPs (Fig. 4I). We were unable to culture SKP-like spheres from the peripheral nerve that innervates the follicle.

Taken together, these results suggest that SKPs represent a neural crest-derived precursor cell population, which can be expanded in culture and are capable of generating glial progeny in response to appropriate cues. In contrast, we could find no such evidence that bone marrow-derived MSCs behave as a neural crest precursor cell with gliogenic potential.

\section{Discussion}

In this study, we have used Wnt1-Cre/Rosa26R ${ }^{\text {LacZ }}$ and Wnt1-Cre/Rosa26R ${ }^{\mathrm{YFP}}$ compound transgenic mice to analyze the developmental rationale by which cells with a Schwann cell phenotype might be generated from precursor cells within bone marrow and skin. These mice permanently express transgenes in cells of neural crest origin and have been previously validated as a method for tracing cells of a neural crest lineage within multiple tissues including bone marrow and skin $(21,23,24,33)$. The combination of LacZ and YFP 
reporters used here allows the determination of transgene expression in colony-forming cells of MSC and SKP cultures and further permits the identification of anatomical distribution of transgene-positive cells in adult tissues. YFP reporter expression also allows FACS analysis and sorting of freshly dissociated live cells (26).

By combining fate mapping with cell sorting and microdissection, we provide a developmental and anatomical rationale for the generation of Schwann cells from SKPs. We demonstrate that facial and back skin-derived SKPs express neural crest reporter transgenes and that SKPs are contained predominantly within the YFP+ fraction of freshly dissociated skin cells from Wnt1-Cre/Rosa26R ${ }^{\text {YFP }}$ mice. Microdissection of facial vibrissal follicles confirms multiple anatomical origins for gliogenic SKPs within epidermal and dermal hair follicles niches. We further demonstrate that both embryonic and adult SKPs express the key neural crest transcription factor Sox10 throughout glial differentiation, during which mature Schwann cell markers such as S100, GFAP, and P0 are expressed. The phenotype of the glial progeny obtained here are consistent with those previously observed. Although we have demonstrated a robust in vitro immunocyotchemical phenotype, we have not demonstrated here in vivo myelinating function of these cells, which is important for their definition as myelinating, rather than nonmyelinating Schwann cells. However, myelination by SKP-derived Schwann cells has been robustly demonstrated by other groups (13).

Since the first description of SKPs in 2001 (17), there has been debate as to whether these cells resemble CNS-derived neurospheres (34) or whether they display properties more in keeping with neural crest precursor cells (3). Our results are broadly consistent with the studies of Miller et al. $(17,32)$ and subsequently Wong et al. (18) and Nagoshi et al. (35), which used a combination of fate mapping, chick injection studies, and functional studies to demonstrate a neural crest origin of SKPs. Our findings are consistent with the majority of studies that show that SKPs express neural crest reporter genes in both facial and back skin $(18,35)$. However, a small number of spheres may be generated from Wnt1-Cre transgene-negative cells derived from back skin.

Previous work has identified the dermal papilla of the adult vibrissal follicle to be a highly enriched, although not exclusive, niche for SKPs $(25,32)$. We demonstrate that unmanipulated, dissociated dermal papilla cells can be differentiated into Schwann cells without intermediate sphere culture, suggesting that endogenous SKPs may possess glial potential.

Recent evidence has suggested a neural crest origin of a subset of adult MSCs (28). However, the definitions of MSCs or marrow stromal cells are broad and encompass a range of cells with potentially varied anatomical and developmental origins.

We therefore asked whether MSCs, specifically derived from adult bone marrow plugs of leg bones, are of neural crest origin. This is an important and relevant starting population, since it reflects the method of harvest of MSCs most commonly used in studies of clinical translation (1,36-39). Using Wnt1-Cre/Rosa26R reporter mice, we were unable to provide evidence that MSCs derived from adult bone marrow of long bones are of neural crest origin.

Specifically, we could find no evidence of transgene expression in the population of colony forming plastic adherent cells derived from adult bone marrow aspirates, which subsequently proliferate to form MSC cultures. Furthermore, colony-initiating cells could not be identified within the $\mathrm{YFP}+$ fraction of freshly isolated Wnt1-Cre/Rosa26R ${ }^{\mathrm{YFP}}$ bone marrow aspirates. In our hands, MSCs derived from bone marrow are not reliably differentiated into cells with a convincing Schwann cell phenotype.

One difficulty in defining the developmental origins of MSCs is the diverse range of methodology that is used to isolate and characterize cells. In these experiments, we have used MSC isolation methodology which has been widely used, in particular in studies that have suggested glial differentiation from MSCs $(1,11)$. This methodology is also similar to that used to isolate MSCs for potential clinical use from adult human subjects (38). It is however possible that the CFU-F are contaminated to some degree by the presence of hematopoietic stem cells. However, parallel cultures reliably expand and generate mesenchymal progeny suggesting that our methodology isolates functional MSCs.

These results do not exclude the possibility that MSCs derived from alternative starting populations such as whole bone preparations are of neural crest origin. We have identified a population of transgene-expressing cells within whole bone preparations, suggesting that there may be cells of neural crest origin within whole bones, although such cells are only rarely found within marrow aspirates. Indeed, studies that have analyzed starting populations derived from crushed bone preparations have demonstrated subsets of MSCs of potential neural crest origin using a P0-Cre neural crest transgenic mouse $(28,35)$.

Taken together, these studies suggest that there may be a neural crest contribution to MSCs earlier in development. However, with regard to MSCs derived from adult long bone aspirates, we are not able to provide evidence of a neural crest origin. Further study of this relationship between the neural crest and mesenchymal precursors in multiple tissues may be of significant interest.

The developmental heterogeneity of subtypes of MSCs and its potential clinical consequences is an important subject for future study. For example, we have recently demonstrated that bone marrow-derived MSCs, when harvested from long bones and characterized according to recognized MSC definitions, do not remyelinate when directly transplanted into a demyelinated lesion (19). Moreover, we demonstrated such MSC grafts are associated with a number of adverse clinical events such as tissue fibrosis and axonal damage. These results contrast with previous studies that demonstrate Schwann cell remyelination by such cell grafts (30). These results caution against the use of undifferentiated MSCs derived from bone marrow plugs as therapeutic remyelinating cells (19). We have previously hypothesized that such discrepancies between centers may be explicable by significant variations in cell culture methodologies that may allow selection of different colony-initiating cells with distinct developmental origins and thus different gliogenic potential (19). We propose that the future systematic study of these differences may help explain the different behavior of these cells in such models of repair and remyelination. These results highlight the need for careful definition of both the protocols for isolation and expansion as well as subsequent definition of developmental origins of cells, particularly when considering their potential clinical applications. 
We conclude that SKPs represent adult tissue-resident neural crest precursors with gliogenic potential. The study of neural crest stem cells has provided important mechanistic insights into a number of human diseases $(40,41)$. The isolation of such cells may therefore be of considerable experimental interest since they may provide mechanistic insight into disorders of human Schwann cell differentiation as well as potentially providing a source of remyelinating cells $(6,10,42)$. An important avenue of further work will be to compare these adult precursor cells with embryonic Schwann cell precursors, which have recently been demonstrated to possess a number of advantages over adult Schwann cells in terms of remyelinating potential (43).

\section{Acknowledgments}

We are very grateful to Nick Greene and Dawn Savery, Neural Development Unit, Institute of Child Health, Great Ormond Street Hospital for generously providing Wnt1Cre/Rosa26R ${ }^{\text {YFP }}$ mice. We are also grateful to Nigel Miller, Department of Pathology, Cambridge University, for expert help with flow cytometry and cell sorting, supported by a grant from the Wellcome Trust. DPJH is supported by a Wellcome Trust Research Training Fellowship, a Brain Entry Scholarship (Guarantors of Brain), and a Raymond and Beverly Sackler Scholarship. KS is supported by grants from the Medical Research Council, European Union Neuropromise, and the Multiple Sclerosis Society. DJH is supported by the British Heart Foundation.

\section{Author Disclosure Statement}

No competing financial interests exist.

\section{References}

1. Akiyama Y, C Radtke and JD Kocsis. (2002). Remyelination of the rat spinal cord by transplantation of identified bone marrow stromal cells. J Neurosci 22:6623-6630.

2. Biernaskie JA, IA McKenzie, JG Toma and FD Miller. (2006). Isolation of skin-derived precursors (SKPs) and differentiation and enrichment of their Schwann cell progeny. Nat Protoc 1:2803-2812.

3. Fernandes KJ, JG Toma and FD Miller. (2008). Multipotent skin-derived precursors: adult neural crest-related precursors with therapeutic potential. Philos Trans R Soc Lond, B, Biol Sci 363:185-198.

4. Baron-Van Evercooren A and W Blakemore. (2004). Remyelination Through Engraftment. Elsevier Science, New York.

5. Jessen KR and R Mirsky. (2005). The origin and development of glial cells in peripheral nerves. Nat Rev Neurosci 6:671-682.

6. Pingault V, N Bondurand, K Kuhlbrodt, DE Goerich, MO Préhu, A Puliti, B Herbarth, I Hermans-Borgmeyer, E Legius, G Matthijs, J Amiel, S Lyonnet, I Ceccherini, G Romeo, JC Smith, AP Read, M Wegner and M Goossens. (1998). SOX10 mutations in patients with Waardenburg-Hirschsprung disease. Nat Genet 18:171-173.

7. Paratore C, C Eichenberger, U Suter and L Sommer. (2002). Sox10 haploinsufficiency affects maintenance of progenitor cells in a mouse model of Hirschsprung disease. Hum Mol Genet 11:3075-3085.

8. Joseph NM, YS Mukouyama, JT Mosher, M Jaegle, SA Crone, EL Dormand, KF Lee, D Meijer, DJ Anderson and SJ Morrison. (2004). Neural crest stem cells undergo multilineage differentiation in developing peripheral nerves to generate endoneurial fibroblasts in addition to Schwann cells. Development 131:5599-5612.
9. Dupin E, S Creuzet and NM Le Douarin. (2006). The contribution of the neural crest to the vertebrate body. Adv Exp Med Biol 589:96-119.

10. Biernaskie J, JS Sparling, J Liu, CP Shannon, JR Plemel, Y Xie, FD Miller and W Tetzlaff. (2007). Skin-derived precursors generate myelinating Schwann cells that promote remyelination and functional recovery after contusion spinal cord injury. J Neurosci 27:9545-9559.

11. Caddick J, PJ Kingham, NJ Gardiner, M Wiberg and G Terenghi. (2006). Phenotypic and functional characteristics of mesenchymal stem cells differentiated along a Schwann cell lineage. Glia 54:840-849.

12. Keilhoff G, A Goihl, K Langnäse, H Fansa and G Wolf. (2006). Transdifferentiation of mesenchymal stem cells into Schwann cell-like myelinating cells. Eur J Cell Biol 85:11-24.

13. McKenzie IA, J Biernaskie, JG Toma, R Midha and FD Miller. (2006). Skin-derived precursors generate myelinating Schwann cells for the injured and dysmyelinated nervous system. J Neurosci 26:6651-6660.

14. Dominici M, K Le Blanc, I Mueller, I Slaper-Cortenbach, F Marini, D Krause, R Deans, A Keating, Dj Prockop and E Horwitz. (2006). Minimal criteria for defining multipotent mesenchymal stromal cells. The International Society for Cellular Therapy position statement. Cytotherapy 8:315-317.

15. Horwitz EM, K Le Blanc, M Dominici, I Mueller, I SlaperCortenbach, FC Marini, RJ Deans, DS Krause and A Keating; the International Society for Cellular Therapy. (2005). Clarification of the nomenclature for MSC: The International Society for Cellular Therapy position statement. Cytotherapy 7:393-395.

16. Raff M. (2003). Adult stem cell plasticity: fact or artifact? Annu Rev Cell Dev Biol 19:1-22.

17. Toma JG, M Akhavan, KJ Fernandes, F Barnabé-Heider, A Sadikot, DR Kaplan and FD Miller. (2001). Isolation of multipotent adult stem cells from the dermis of mammalian skin. Nat Cell Biol 3:778-784.

18. Wong CE, C Paratore, MT Dours-Zimmermann, A Rochat, $\mathrm{T}$ Pietri, U Suter, DR Zimmermann, S Dufour, JP Thiery, D Meijer, F Beermann, Y Barrandon and L Sommer. (2006). Neural crestderived cells with stem cell features can be traced back to multiple lineages in the adult skin. J Cell Biol 175:1005-1015.

19. Hunt DP, KA Irvine, DJ Webber, DA Compston, WF Blakemore and S Chandran. (2008). Effects of direct transplantation of multipotent mesenchymal stromal/stem cells into the demyelinated spinal cord. Cell Transplant 17:865-873.

20. Phinney DG and DJ Prockop. (2007). Concise review: mesenchymal stem/multipotent stromal cells: the state of transdifferentiation and modes of tissue repair--current views. Stem Cells 25:2896-2902.

21. Jiang X, S Iseki, RE Maxson, HM Sucov and GM Morriss-Kay. (2002). Tissue origins and interactions in the mammalian skull vault. Dev Biol 241:106-116.

22. Danielian PS, D Muccino, DH Rowitch, SK Michael and AP McMahon. (1998). Modification of gene activity in mouse embryos in utero by a tamoxifen-inducible form of Cre recombinase. Curr Biol 8:1323-1326.

23. Szeder V, M Grim, Z Halata and M Sieber-Blum. (2003). Neural crest origin of mammalian Merkel cells. Dev Biol 253: 258-263.

24. Jiang X, DH Rowitch, P Soriano, AP McMahon and HM Sucov. (2000). Fate of the mammalian cardiac neural crest. Development 127:1607-1616.

25. Hunt DP, PN Morris, J Sterling, JA Anderson, A Joannides, C Jahoda, A Compston and S Chandran. (2008). A highly enriched niche of precursor cells with neuronal and glial potential within the hair follicle dermal papilla of adult skin. Stem Cells 26:163-172.

26. Srinivas S, T Watanabe, CS Lin, CM William, Y Tanabe, TM Jessell and F Costantini. (2001). Cre reporter strains produced 
by targeted insertion of EYFP and ECFP into the ROSA26 locus. BMC Dev Biol 1:4.

27. Peister A, JA Mellad, BL Larson, BM Hall, LF Gibson and DJ Prockop. (2004). Adult stem cells from bone marrow (MSCs) isolated from different strains of inbred mice vary in surface epitopes, rates of proliferation, and differentiation potential. Blood 103:1662-1668.

28. Takashima Y, T Era, K Nakao, S Kondo, M Kasuga, AG Smith and S Nishikawa. (2007). Neuroepithelial cells supply an initial transient wave of MSC differentiation. Cell 129:1377-1388.

29. Glennie S, I Soeiro, PJ Dyson, EW Lam and F Dazzi. (2005). Bone marrow mesenchymal stem cells induce division arrest anergy of activated T cells. Blood 105:2821-2827.

30. Akiyama Y, C Radtke, O Honmou and JD Kocsis. (2002). Remyelination of the spinal cord following intravenous delivery of bone marrow cells. Glia 39:229-236.

31. Paratore C, DE Goerich, U Suter, M Wegner and L Sommer. (2001). Survival and glial fate acquisition of neural crest cells are regulated by an interplay between the transcription factor Sox10 and extrinsic combinatorial signaling. Development 128:3949-3961.

32. Fernandes KJ, IA McKenzie, P Mill, KM Smith, M Akhavan, F Barnabé-Heider, J Biernaskie, A Junek, NR Kobayashi, JG Toma, DR Kaplan, PA Labosky, V Rafuse, CC Hui and FD Miller. (2004). A dermal niche for multipotent adult skin-derived precursor cells. Nat Cell Biol 6:1082-1093.

33. Sieber-Blum M, M Grim, YF Hu and V Szeder. (2004). Pluripotent neural crest stem cells in the adult hair follicle. Dev Dyn 231:258-269.

34. Belicchi M, F Pisati, R Lopa, L Porretti, F Fortunato, M Sironi, M Scalamogna, EA Parati, N Bresolin and Y Torrente. (2004). Human skin-derived stem cells migrate throughout forebrain and differentiate into astrocytes after injection into adult mouse brain. J Neurosci Res 77:475-486.

35. Nagoshi N, S Shibata, Y Kubota, M Nakamura, Y Nagai, E Satoh, S Morikawa, Y Okada, Y Mabuchi, H Katoh, S Okada, K Fukuda, T Suda, Y Matsuzaki, Y Toyama and H Okano. (2008). Ontogeny and multipotency of neural crest-derived stem cells in mouse bone marrow, dorsal root ganglia, and whisker pad. Cell Stem Cell 2:392-403.

36. Bang OY, JS Lee, PH Lee and G Lee. (2005). Autologous mesenchymal stem cell transplantation in stroke patients. Ann Neurol 57:874-882.
37. Gerdoni E, B Gallo, S Casazza, S Musio, I Bonanni, E Pedemonte, R Mantegazza, F Frassoni, G Mancardi, R Pedotti and A Uccelli. (2007). Mesenchymal stem cells effectively modulate pathogenic immune response in experimental autoimmune encephalomyelitis. Ann Neurol 61:219-227.

38. Le Blanc K, I Rasmusson, B Sundberg, C Götherström, M Hassan, M Uzunel and O Ringdén. (2004). Treatment of severe acute graft-versus-host disease with third party haploidentical mesenchymal stem cells. Lancet 363:1439-1441.

39. Le Blanc K and O Ringdén. (2006). Mesenchymal stem cells: properties and role in clinical bone marrow transplantation. Curr Opin Immunol 18:586-591.

40. Buchstaller J, L Sommer, M Bodmer, R Hoffmann, U Suter and N Mantei. (2004). Efficient isolation and gene expression profiling of small numbers of neural crest stem cells and developing Schwann cells. J Neurosci 24:2357-2365.

41. Iwashita T, GM Kruger, R Pardal, MJ Kiel and SJ Morrison. (2003). Hirschsprung disease is linked to defects in neural crest stem cell function. Science 301:972-976.

42. Zawadzka M and RJ Franklin. (2007). Myelin regeneration in demyelinating disorders: new developments in biology and clinical pathology. Curr Opin Neurol 20:294-298.

43. Woodhoo A, V Sahni, J Gilson, A Setzu, RJ Franklin, WF Blakemore, R Mirsky and KR Jessen. (2007). Schwann cell precursors: a favourable cell for myelin repair in the Central Nervous System. Brain 130(Pt 8):2175-2185.

Address correspondence to: Dr. David Hunt Anne Maclaren Laboratory for Regenerative Medicine MRC Centre for Stem Cell Biology and Regenerative Medicine

Forvie Site

University of Cambridge Cambridge CB2 2PY United Kingdom

E-mail:dpjh2@cam.ac.uk

Received for publication September 22, 2009

Accepted after revision January 26, 2010

Prepublished on Liebert Instant Online January 26, 2010 
